\title{
Survival to hospital discharge and neurological outcomes with targeted temperature management after in-hospital cardiac arrest: a systematic review and meta-analysis
}

\author{
Li Yin ${ }^{1 \wedge}$, Deng Xie ${ }^{2}$, Daofen $\mathrm{He}^{1}$, Zhiwei Chen ${ }^{1}$, Yunjie Guan ${ }^{1}$, Jing Wang ${ }^{1}$, Zhihong Lin $^{1 \wedge}$ \\ ${ }^{1}$ Emergency Department, the First Affiliated Hospital of Fujian Medical University, Fuzhou, China; ${ }^{2}$ Department of Cardiovascular Surgery, Fujian \\ Provincial Hospital, Shengli Clinical College of Fujian Medical University, Fuzhou, China \\ Contributions: (I) Conception and design: Z Lin, L Yin; (II) Administrative support: Z Lin; (III) Provision of study materials or patients: L Yin, D Xie; \\ (IV) Collection and assembly of data: L Yin, D Xie, D He; (V) Data analysis and interpretation: L Yin, Z Chen, Y Guan; (VI) Manuscript writing: All \\ authors; (VII) Final approval of manuscript: All authors. \\ Correspondence to: Zhihong Lin. Emergency Department, the First Affiliated Hospital of Fujian Medical University, Fuzhou 350005, China. \\ Email: fzlinzh@163.com.
}

Background Multiple randomized controlled trials have shown that targeted temperature management (TTM) has favorable effects in out-of-hospital cardiac arrest. However, the benefit of TTM in patients with in-hospital cardiac arrest (IHCA) remains to be verified.

Methods: The PubMed, Cochrane Library, and EMBASE databases were searched for clinical studies with the primary outcomes of survival to hospital discharge and neurological outcomes. Neurological outcomes were evaluated by the categorical scale of cerebral function (CPC); a score of 1-2 points was considered neurologically good, and a score of 3-5 points was considered a poor outcome. Revman 5.3 and Stata 14 software with the random effects model were used for analysis. $\mathrm{P}<0.05$ was considered statistically significant. Results: Six retrospective controlled studies with a total of 14,607 patients (TTM group: 1,845, control group: 12,762) were included and analyzed. There were no statistically significant differences between the two groups in survival to hospital discharge [odds ratio $(\mathrm{OR})=1.02,95 \% \mathrm{CI}: 0.77-1.35, \mathrm{P}=0.89, \mathrm{I}^{2}=47 \%$ ] or favorable neurological outcomes ( $\mathrm{OR}=1.06,95 \% \mathrm{CI}$ : 0.56-2.02, $\left.\mathrm{P}=0.85, \mathrm{I}^{2}=79 \%\right)$. After excluding patients with non-shockable initial rhythms, TTM did not show any significant improvement in survival to hospital discharge. Subgroup analysis was performed according to the sample size. No significant improvement was observed between the two groups in terms of survival to hospital discharge or neurological outcome.

Discussion: In this meta-analysis, the effects of TTM on discharge survival and neurological prognosis were evaluated by studying the results of IHCA in 14,607 patients. We found that the TTM did not improve survival and neurological function in discharged patients. Our results showed that the sample size discrepancy had a large effect on the heterogeneity; to address this, subgroup analyses were performed according to the different sample sizes. However, TTM treatment in different sample size subgroups showed no significant effect on survival to hospital discharge. Moreover, in the large sample size subgroup, therapeutic hypothermia was associated with increased unfavorable neurological outcome compared with no hypothermia.

Keywords: In-hospital cardiac arrest (IHCA); targeted temperature management (TTM); survival to hospital discharge; neurological outcome; meta-analysis

Submitted Oct 29, 2021. Accepted for publication Dec 30, 2021.

doi: $10.21037 /$ apm-21-3403

View this article at: https://dx.doi.org/10.21037/apm-21-3403

^ ORCID: Li Yin, 0000-0002-8674-8452; Zhihong Lin, 0000-0002-3967-6863. 


\section{Introduction}

The incidence of sudden cardiac arrest has become an increasingly prevalent global health problem due to population growth and increasing cardiovascular deaths worldwide (1), with an annual rate of $50-100$ per 100,000 people (2). In contrast to death without resuscitation, in-hospital cardiac arrest (IHCA) is generally defined as the loss of circulation prompting resuscitation with chest compressions, defibrillation, or a combination of both. In the United States, more than 290,000 adults develop IHCA each year, with a hospital discharge survival rate of approximately $25 \%$, and surviving patients mostly present with complications (3).

Targeted temperature management (TTM) is strictly temperature controlled, with a core body temperature after cardiac arrest between 33 and $36^{\circ} \mathrm{C}$. It includes three aspects: induction of mild hypothermia, mild hypothermia for pre-hospital rescue, and avoidance of hyperthermia. According to the guidelines for cardiopulmonary resuscitation (CPR) issued by the American Heart Association (AHA), therapy needs to be started immediately upon admission and the target temperature maintained for $4 \mathrm{~h}$. The target temperature is then maintained for $24 \mathrm{~h}$ and rewarmed to $37^{\circ} \mathrm{C}$ (at a rate of $0.25^{\circ} \mathrm{C}$ per hour over 12-16 h, not exceeding a rate of $\left.0.5^{\circ} \mathrm{C} / \mathrm{h}\right)(4,5)$.

Cerebral reperfusion injury occurs when cerebral blood flow is restored after cardiac arrest and resuscitation. Increased intracellular levels of glutamate, an excitatory neurotransmitter released from presynaptic terminals, activate ion-channel complexes that cause calcium to shift from the extracellular to the intracellular fluid, leading to the accumulation of oxygen free radicals and the activation of degradative enzymes. In addition, cerebral hemodynamics may remain abnormal after resuscitation from cardiac arrest. Hypothermia not only reduces cerebral metabolic rate for oxygen but also reduces the glutamate level and the subsequent production of oxygen free radicals. Therefore, hypothermia improves neurological outcomes in patients who are comatose after resuscitation from out-ofhospital cardiac arrest.

In 2002, two landmark randomized controlled trials (RCTs) were published simultaneously in the New England Fournal of Medicine. The hypothermia after cardiac arrest (HACA) research team included 275 patients resuscitated after out-of-hospital cardiac arrest. It was found that the good outcome rate of the nervous system increased by $15.8 \%$ in the hypothermia group compared with the normal temperature group (55.2\% vs. 39.4\%) (6,7). In 2003, the International Resuscitation Consortium (ILCOR) issued recommendations on post-resuscitation hypothermia management. For patients with out-of-hospital cardiac arrest, whose initial heart rate is ventricular fibrillation, and there is no response after recovery of spontaneous circulation (ROSC), it is recommended to cool to $32-34{ }^{\circ} \mathrm{C}$ for 12-24 h. This therapy is also applicable to other types of cardiac arrest or IHCA (8).

In 2005, the AHA wrote this protocol into the guidelines, and the proposal was adopted until 2010 (9). Relevant studies have shown that patients with IHCA have the same benefits as patients with out-of-hospital cardiac arrest treated with TTM; however, most studies believe that mild hypothermia treatment after hospital cardiac arrest does not provide significant benefits to patients, and may even be harmful (10). To date, relevant RCTs are scant. Therefore, whether to carry out therapeutic hypothermia treatment for patients with cardiac arrest in hospital is still controversial.

Many previous similar meta-analyses have focused on survival and neurological outcomes of out-of-hospital sudden cardiac arrest or simultaneous analysis of target temperature management after in-hospital sudden cardiac arrest. Articles that only analyze survival and neurological outcomes of target temperature management after inhospital sudden cardiac arrest are very rare. Therefore, we hypothesize that the use of target temperature management after in-hospital sudden cardiac arrest is not only not beneficial to discharge survival and neurological outcomes, but even harmful.

The purpose of this meta-analysis was to systematically analyze the discharge survival and neurological outcomes in patients treated with TTM after restoring autonomic circulation following IHCA. We present the following article in accordance with the MOOSE reporting checklist (available at https://apm.amegroups.com/article/ view/10.21037/apm-21-3403/rc).

\section{Methods}

\section{Data sources and searches}

We performed a literature search of the PubMed, EMBASE, and Cochrane databases from the date of inception of the database to December 2020, using the following search terms: "Therapeutic Hypothermia" or "Hypothermia, Therapeutic" or "Targeted Temperature Management" or "Targeted Temperature Managements, Induced 


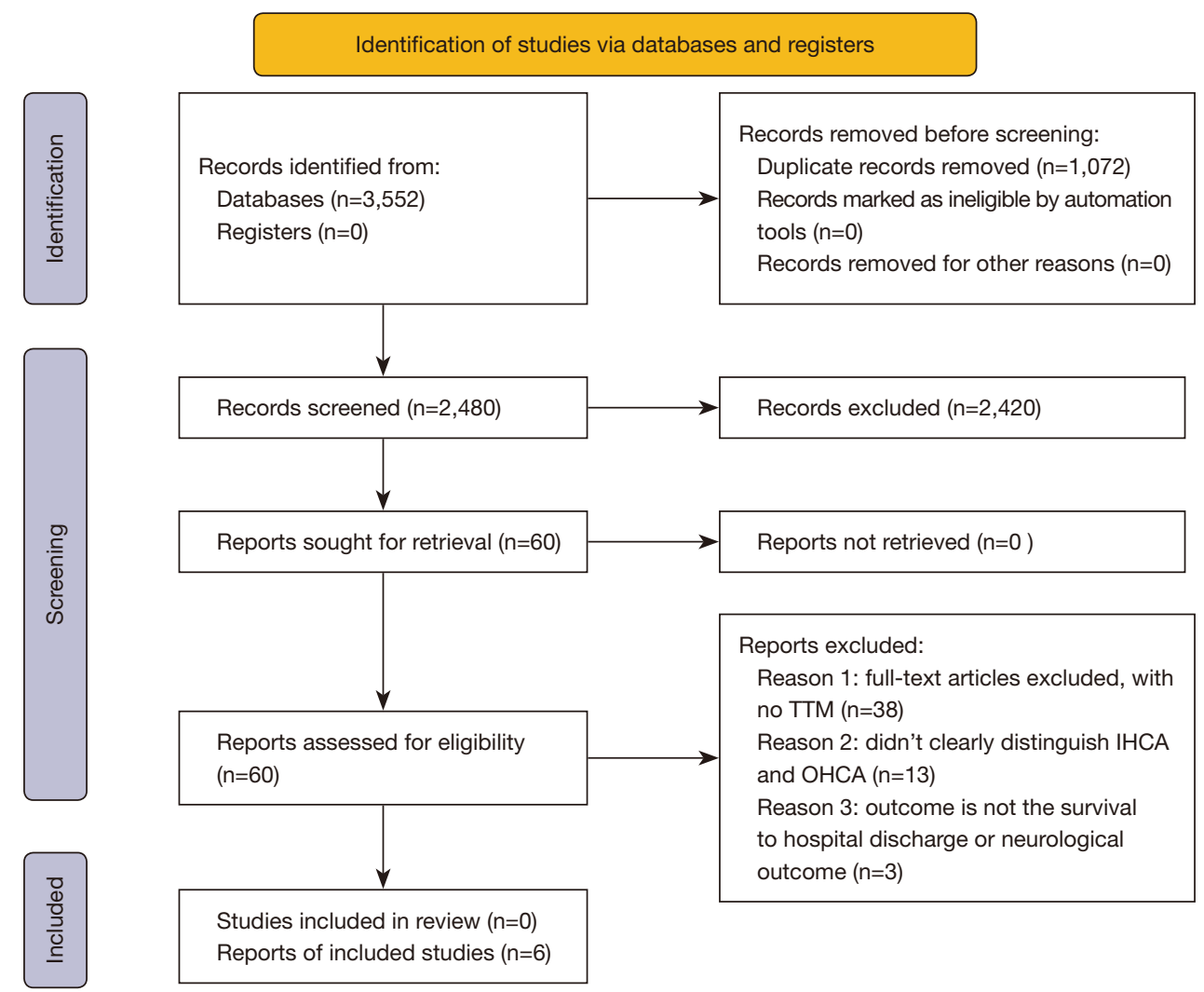

Figure 1 Study selection flow chart. TTM, targeted temperature management; IHCA, in-hospital cardiac arrest; OHCA, out-of-hospital cardiac arrest.

Hypothermia" or "Moderate Hypothermia, Induced" or "Induced Moderate Hypothermia" or "Induced Moderate Hypothermias" or "Moderate Hypothermias, Induced" or "Mild Hypothermia, Induced" or "Induced Mild Hypothermia" or "Induced Mild Hypothermias" or "Mild Hypothermias, Induced" and "Arrest, Heart" or "Cardiac Arrest" or "Arrest, Cardiac" or "Asystole" or "Asystoles" or "Cardiopulmonary Arrest" or "Arrest, Cardiopulmonary".. The review questions were developed according to the population, intervention, comparison, and outcome (PICO) protocol, as specified in Appendix A. The criteria for inclusion were as follows: (I) all patients aged $\geq 18$ years; (II) patients who suffered cardiac arrest in the hospital; (III) patients who achieved a successful return of spontaneous circulation; (IV) studies involving at least 10 or more patients who underwent TTM; and (V) all trials meeting $>4$ criteria of appeal (including observational studies, cohort studies, case-control studies, etc.) and comparing TTM in hospitalized cardiac arrest patients with a control group for survival to hospital discharge and neurological outcomes.

\section{Study selection}

Two authors (Li Yin and Deng Xie) independently conducted the literature search and screened the studies, and any disagreements were resolved by the third author (Zhihong Lin). The study selection flow chart is shown in Figure 1. The preliminary search yielded 3,552 articles. Of these, 1,072 articles were duplicated, and we excluded 3,492 articles as they didn't meet the inclusion criteria. A detailed review of the titles and abstracts of the 60 remaining studies resulted in exclusion of 38 studies due to non-adherence to the inclusion criteria. Twenty-two studies were selected for further review, and the full manuscripts of these papers were read and their references lists were assessed for further publications. Sixteen studies were excluded because the outcomes of the TTM group versus the control group were not clearly determined and because patients with IHCA and out-of-hospital cardiac arrest were not distinguished. Finally, six retrospective cohort studies that utilized data from patients with IHCA were included in this meta-analysis. 
Table 1 NOS scale

\begin{tabular}{|c|c|c|c|c|}
\hline Studies & Selection & Comparability & Outcome & Total score \\
\hline Wang JC, 2020 & 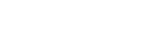 & 论㑔 & $i$ & 7 \\
\hline Kory P, 2012 & 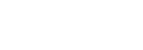 & 弥弥 & 论场 & 8 \\
\hline Engsig M, 2016 & 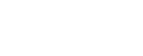 & 㑔柁 & 论访 & 8 \\
\hline Dankiewicz J, 2014 & 论放放放 & 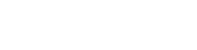 & 论论㑔 & 8 \\
\hline Perman SM, 2015 & 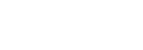 & is & 论㘧访 & 8 \\
\hline
\end{tabular}

NOS, Newcastle-Ottawa-Scale.

\section{Data extraction}

Two reviewers (YL and $\mathrm{XD}$ ) independently extracted the data for meta-analysis, and discrepancies were resolved by the third reviewer (Zhihong Lin) and by consensus. We extracted information including the year of study, sample size, ethnicity, whether it was multi-center data, discharge survival of the TTM and control groups, and the neurological outcomes of the TTM and control groups. The remaining indexes such as TTM method, time and temperature could not be extracted because of incomplete data.

\section{Grade appraisal}

The quality of the evidence was judged by two authors (YL, $\mathrm{XD})$ who independently, assessed the articles for limitations (risk of bias), indirectness, pre- and post-inconsistency, and imprecision according to the recommendations (grade of assessment, formulation, and evaluation). Given that the included literature were all observational studies, the quality of the articles was evaluated according to the NewcastleOttawa-Scale (NOS) scale, which was greater than or equal to six stars, and we considered the quality of the articles to be high-quality articles. Of the six included studies, three scored 7 stars, two scored 8 stars, and one scored 9 stars. All of the included studies were above 6 stars (Table 1).

\section{Statistical analysis}

Using data from the six included studies, we performed meta-analyses to assess the rates of discharge survival and neurological outcomes of IHCA patients in the control and TTM groups. Additionally, we performed subgroup analyses to analyze survival to hospital discharge in the TTM group after excluding data for non-shockable initial rhythms. Sample content was discriminated to assess the impact of sample content on hospital discharge survival and neurological outcomes. The odds ratios (ORs) for each trial were calculated. The Mantel-Haenzel method was utilized to calculate the pooled OR for fixed effect, and the DerSimonian and Laird model was used for randomeffects. The examination of heterogeneity by L'Abbe and Galbraith plots. Sensitivity analysis was performed using the random effects model method and single study impact plot. We used the $\mathrm{I}^{2}$ statistic to assess for heterogeneity, which was considered as low, moderate, or high for $25 \%, 50 \%$, and $75 \%$, respectively. When heterogeneity was significant $\left(\mathrm{I}^{2}>50 \%\right)$, inconsistency across studies was defined as severe. Publication bias was assessed by visual inspection of the funnel plot. We calculated the $95 \%$ confidence intervals (CIs) for the different variables. $\mathrm{P}<0.05$ was considered statistically significant. Meta-analysis was performed using the REVMAN 5.3 (Cochrane, UK) and Stata 14 (Statacorp, Texas, USA) software programs.

\section{Ethics statement}

Ethical approval: since all analyses were based on previously published studies, ethical approval was not required for this meta-analysis.

\section{Results}

\section{Study characteristics}

Six retrospective cohort studies were included in this metaanalysis (11-16). The extracted data are shown in Figure 1. One study applied TTM in patients with IHCA and outof-hospital cardiac arrest, while the remaining five studies treated patients with TTM after IHCA. All six studies were 
Table 2 Extracted data from the selected studies

\begin{tabular}{ccccccccccccc}
\hline Year & Author & TTMs & Controls & TTMNg & ControlNs & $\begin{array}{c}\text { TTM } \\
\text { content }\end{array}$ & $\begin{array}{c}\text { NTTM } \\
\text { content }\end{array}$ & $\begin{array}{c}\text { Study } \\
\text { population } \\
\text { size }\end{array}$ & $\begin{array}{c}\text { Race } \\
\text { Center }\end{array}$ \\
\hline 2016 & Chan PS & $417 / 1,524$ & $1,084 / 3,714$ & $246 / 1,443$ & $725 / 3,529$ & 1,524 & 3,714 & 5,238 & - & Multi \\
2020 & Wang JC & $11 / 26$ & $81 / 154$ & $1 / 26$ & $57 / 154$ & 26 & 154 & 262 & - & Single \\
2013 & Nichol G & $58 / 214$ & $2,454 / 8,102$ & $17 / 91$ & $1,591 / 8,102$ & 214 & 8,102 & 8,316 & - & Multi \\
2016 & Wang CH & $9 / 22$ & $135 / 656$ & $7 / 22$ & $53 / 656$ & 22 & 656 & 678 & - & Single \\
2012 & Kory P & $5 / 17$ & $5 / 16$ & $4 / 17$ & $5 / 16$ & 17 & 16 & 33 & - & Single \\
2015 & Perman SM & $12 / 42$ & $21 / 120$ & $9 / 42$ & $16 / 120$ & 42 & 120 & 162 & - & Multi \\
\hline
\end{tabular}

TTM, targeted temperature management; TTMs, TTM survival to hospital discharge; Controls: Control group survival to hospital discharge; TTMNg, TTM good neurological outcome; ControlNs, Control group good neurological outcome; NTTM, no targeted temperature management.

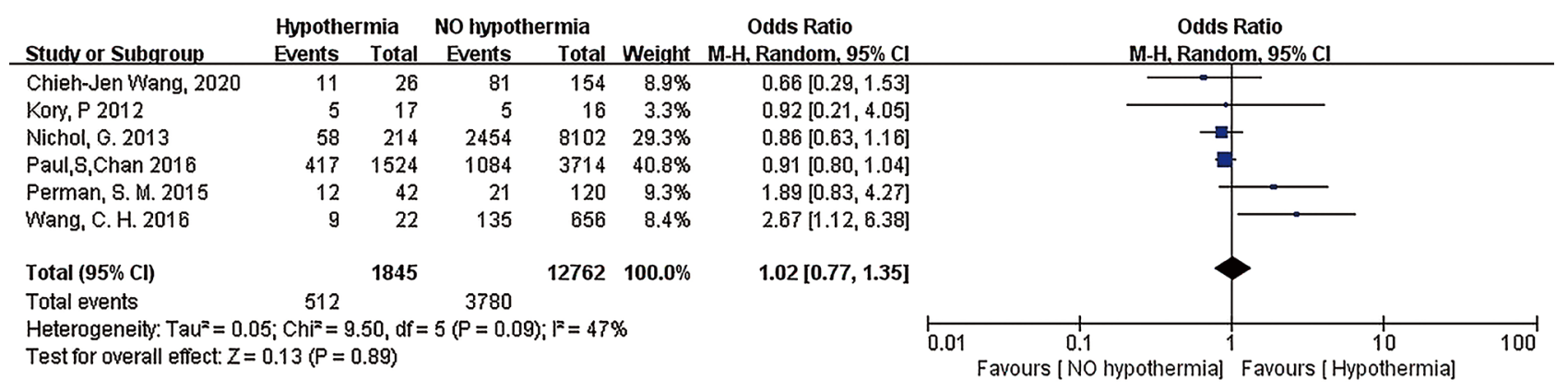

Figure 2 Survival to hospital discharge.

retrospective. Three of these studies applied the Get With The Guidelines (GWTG) resuscitation registry scale (17) to collect multi-center data $(11,13,14)$, and the other three studies were all single-center studies (Table 2).

\section{Search results}

A total of six studies were included in the analysis of survival to hospital discharge, which contained 14,607 patients (TTM group: 1,845; control group: 12,762). No significant improvement for the survival to hospital discharge (OR $=1.02$, 95\% CI: 0.77-1.35, Figure 2) was observed in the TTM group compared to the control group. There was moderate heterogeneity between the studies $(\mathrm{P}=0.89$, $\left.I^{2}=47 \%\right)$.

A total of six studies were included in the analysis of good neurological outcomes, which contained 14,215 patients (TTM group: 1,641; control group: 12,574). No significant improvement was observed in the good neurological outcomes of the therapeutic hypothermia group compared with the control group ( $\mathrm{OR}=1.06,95 \%$ CI: 0.56-2.02, Figure 3). A high level of inter-study heterogeneity was observed $\left(\mathrm{P}=0.85, \mathrm{I}^{2}=79 \%\right)$.

\section{Shockable initial rhythms subgroup analysis}

For subgroup analysis, after excluding patients with non-shockable initial rhythms, two studies with a total of 1,327 patients were included (TTM group: 428; control group: 899). Compared to the controls, TTM treatment did not significantly improve the survival to hospital discharge of IHCA patients (OR $=0.89,95 \%$ CI: $0.71-1.13$, Figure $\mathrm{S} 1)$. The $\mathrm{I}^{2}$ statistic indicated a very low level of heterogeneity between the studies $\left(\mathrm{P}=0.35, \mathrm{I}^{2}=0 \%\right)$.

\section{Different sample size subgroup analysis}

Due to the large sample size gap, subgroup analyses were performed according to sample sizes; small samples were defined as those with TTM $<50$, while large samples were 


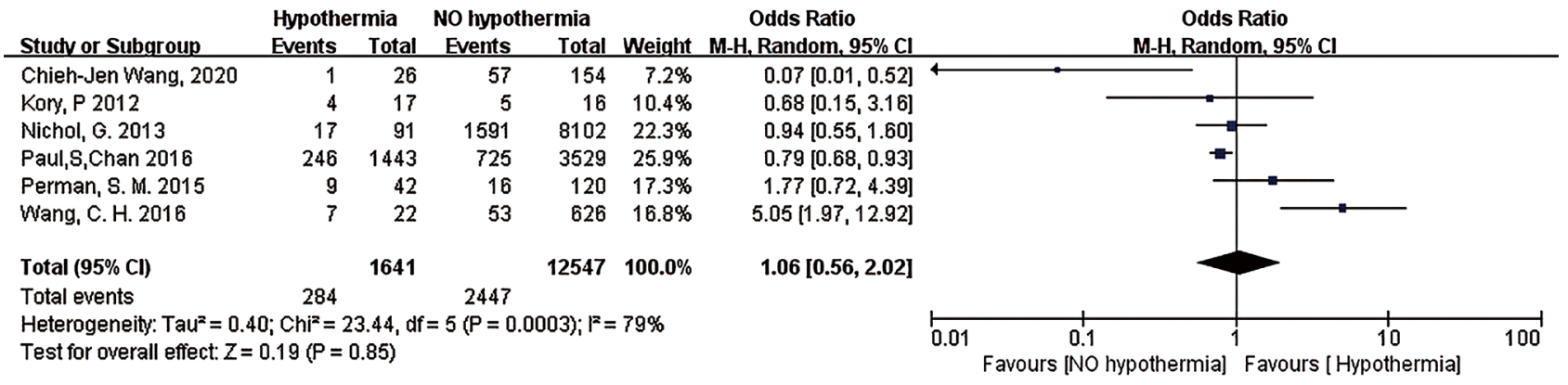

Figure 3 Good neurological outcome.

defined as those with TTM $>50$.

For hospital discharge survival, the small sample size subgroup analysis included four studies with a total of 1,327 patients (TTM group: 116; control group: 1,019). No significant improvement was observed in the TTM group compared with control group (OR $=0.82,95 \%$ CI: $0.17-$ 3.99, Figure S2), A high level of study heterogeneity was observed $\left(\mathrm{I}^{2}=90 \%, \mathrm{P}=0.81\right)$. The large sample size subgroup analysis included two studies with a total of 13,599 patients (TTM group: 1,783; control group: 11,816). Likewise, there was no significant improvement in hospital discharge survival in the TTM group compared with the control group $(\mathrm{OR}=0.90,95 \% \mathrm{CI}$ : 0.80-1.02, Figure S2), and a low level of study heterogeneity was observed $\left(\mathrm{I}^{2}=0 \%, \mathrm{P}=0.11\right)$.

For neurological outcome, the small sample size subgroup included four studies with a total of 1,053 patients (TTM group: 107; control group: 946). No significant improvement in neurological outcome was observed in the TTM group compared with control group ( $\mathrm{OR}=0.97$, 95\% CI: 0.19-5.03, Figure S3), and a high level of study heterogeneity was observed $\left(\mathrm{I}^{2}=86 \%, \mathrm{P}=0.97\right)$. The large sample size subgroup analysis included two studies with a total of 13,165 patients (TTM group: 1,534; control group: 11,631). Compared with the control group, treatment with TTM the aggravated unfavorable neurological outcomes $(\mathrm{OR}=0.81,95 \%$ CI: 0.69-0.94, Figure S3), and a low level of study heterogeneity was observed $\left(\mathrm{I}^{2}=0 \%, \mathrm{P}=0.006\right)$.

\section{Heterogeneity and sensitivity analysis}

We conducted subgroup analysis and found that the sample size discrepancy had a large effect on the heterogeneity (as detailed in Figures S2,S3). The examination of heterogeneity by L'Abbe and Galbraith plots revealed that three articles resulted in a large heterogeneity in the early discharge survival group, and two articles resulted in a large heterogeneity in the good neurological outcome group (for details, see Figures S4-S9). We speculated that this might be because of the sample size discrepancy, and performed further investigation to verify this. Sensitivity analysis was performed using the random effects model method and single study impact plot (for details, see Figures S10-S13). Publication bias was assessed by visual inspection of the funnel plots, and found that our results may have publication bias, but due to the small number of included articles and high heterogeneity, it may affect the outcome (as shown in Figure S14).

\section{Discussion}

In this meta-analysis, the effects of TTM on discharge survival and neurological prognosis were evaluated by studying the results of IHCA in 14,607 patients. We found that TTM treatment did not improve survival and neurological function in discharged patients.

During cardiac arrest, cerebral perfusion and oxygen supply are impaired, and the consumption of glucose and energy reserves is increased. In addition, free radicals may be produced when cerebral perfusion is restored after restoration of spontaneous circulation, which may lead to further nerve injury and even loss of normal cell function and integrity. TTM treatment following cardiac arrest reduces the oxygen demand of the brain by decreasing its metabolic rate. In addition, TTM can reduce the production of oxygen free radicals and subsequent cell damage (18-20).

Although guidelines recommend the same treatment as patients with out-of-hospital cardiac arrest, TTM should be performed if patients with in IHCA are still unconscious after ROSC. However, there is presently a lack of relevant RCT research, and therefore, whether patients with IHCA should receive therapeutic hypothermia remains 
controversial.

A previous study comparing the use of TTM treatment after IHCA and out-of-hospital cardiac arrest showed that patients with IHCA were usually described as worse, significantly higher comorbidity, and higher inherent risk of death. However, there was no significant difference in the cardiovascular complications between the two groups. This may be because no difference was observed in the comorbidities before arrest, and IHCA patients had higher bystander cardiopulmonary resuscitation (CPR) frequency and shorter time to ROSC. Also, compared with out-ofhospital cardiac arrest, IHCA was identified earlier, the time to start advanced life support was shorter, the treatment of potential reversible causes was faster, and the quality of emergency resuscitation, ventilation, and CPR was higher. Despite the increased incidence of initial arrhythmias in the IHCA group, there was no difference in discharge survival and neurological outcomes between patients with out-ofhospital cardiac arrest and IHCA treated with TTM (21), which is consistent with our findings.

Therapeutic hypothermia improves overall survival and good neurological outcomes in patients with out-of-hospital cardiac arrest due to a shockable initial heart rhythm (ventricular fibrillation or pulseless ventricular tachycardia); however, more than $80 \%$ of patients with IHCA present with a non-shockable initial heart rhythm (ventricular asystole or pulseless electrical activity), leading to an uncertain benefit of their targeted temperature management (TTM). We therefore performed subgroup analysis and found that TTM was not significantly associated with improvements of survival to hospital discharge and good neurological outcomes in IHCA patients after removing all non-shockable initial heart rhythms cases.

Moreover, our results also showed that the sample size discrepancy had a large effect on the heterogeneity. To address this, subgroup analyses were performed according to the different sample sizes. However, to our disappointment, TTM treatment in different sample size subgroups had no significant effect on survival to hospital discharge. Furthermore, in the large sample size subgroup, therapeutic hypothermia was associated with increased unfavorable neurological outcomes compared with no hypothermia.

Contrary to the beneficial effects on patients with outof-hospital cardiac arrest, treatment with TTM did not show any improvement of survival to hospital or favorable neurological outcomes in IHCA patients, even in those with a shockable initial heart rhythm. This contradiction may be due to the different condition between in-hospital and outof-hospital. Faster response times and a higher quality of emergency resuscitation potentially limited the theorized benefit of therapeutic hypothermia to reduce free radicalmediated reperfusion injury from anoxic brain injury in IHCA patients. Taken together, based on the results of our meta-analysis and those of previous studies, it might be not the time to introduce therapeutic hypothermia to patients with IHCA.

Because stroke and cardiac arrest have a similar pathophysiological process after brain injury, The study of therapeutic hypothermia for ischemic stroke found that: early treatment leads to better outcomes; prolonged hypothermia is unnecessary; light hypothermia $\left(33-35^{\circ} \mathrm{C}\right)$ is probably sufficient, with negative effects associated with lower temperatures below $30^{\circ} \mathrm{C}$. Therefore, TTM has a synergistic effect in the treatment of stroke patients.

Given the large heterogeneity among studies of good neurological outcomes at discharge, we applied the random effects model method and the single study effect method when conducting the sensitivity analysis, and the random effects model method had little effect on the heterogeneity. When removing single studies, the heterogeneity was significantly reduced after removing Wang CH, 2016 (15) $\left(\mathrm{I}^{2}=47 \% \rightarrow \mathrm{I}^{2}=0 \%\right)$ in the survival study at hospital discharge (as detailed in Figure S13). In a neurological outcome study, the heterogeneity was also significantly reduced after removing Wang $\mathrm{CH}, 2016(15)\left(\mathrm{I}^{2}=82 \% \rightarrow \mathrm{I}^{2}=56 \%\right)$. A possible reason for this was that the sample size was small in the experimental group, and the lower positive rate resulted in significantly higher heterogeneity among the studies.

Our study has some limitations that should be noted. Firstly, all of the included studies were retrospective and observational studies, so the overall evidence quality of predictive variables was low. However, the observational design is the standard for prognostic studies. Secondly, the number of included studies is small, and the sample size gap between the control and experimental groups was large, which may lead to significant heterogeneity. Next, for neurological outcomes in patients with shockable initial rhythms, analysis could not be performed due to the paucity of data. Lastly, the meta-analysis was based on pooled research data, which only evaluated the univariate association between intervention factors and patient outcomes. However, there may be more confounding factors, such as specific cryotherapy methods, time, etc. To adjust for major confounders, a meta-analysis of individual 
patient data (IPD) is needed.

\section{Conclusions}

This meta-analysis showed that TTM in patients with IHCA does not improve discharge survival or neurological prognosis. However, more randomized controlled studies are needed to confirm these findings.

\section{Acknowledgments}

Funding: This study was supported by grants from the Science and Technology Foundation of Fujian Province (2021Y0014).

\section{Footnote}

Reporting Checklist: The authors have completed the MOOSE reporting checklist. Available at https://apm. amegroups.com/article/view/10.21037/apm-21-3403/rc

Conflicts of Interest: All authors have completed the ICMJE uniform disclosure form (available at https://apm. amegroups.com/article/view/10.21037/apm-21-3403/coif). The authors have no conflicts of interest to declare.

Ethical Statement: The authors are accountable for all aspects of the work in ensuring that questions related to the accuracy or integrity of any part of the work are appropriately investigated and resolved.

Open Access Statement: This is an Open Access article distributed in accordance with the Creative Commons Attribution-NonCommercial-NoDerivs 4.0 International License (CC BY-NC-ND 4.0), which permits the noncommercial replication and distribution of the article with the strict proviso that no changes or edits are made and the original work is properly cited (including links to both the formal publication through the relevant DOI and the license). See: https://creativecommons.org/licenses/by-nc-nd/4.0/.

\section{References}

1. Roth GA, Forouzanfar MH, Moran AE, et al.

Demographic and epidemiologic drivers of global cardiovascular mortality. N Engl J Med 2015;372:1333-41.

2. Berdowski J, Berg RA, Tijssen JG, et al. Global incidences of out-of-hospital cardiac arrest and survival rates:
Systematic review of 67 prospective studies. Resuscitation 2010;81:1479-87.

3. Andersen LW, Holmberg MJ, Berg KM, et al. In-Hospital Cardiac Arrest: A Review. JAMA 2019;321:1200-10.

4. Duff JP, Topjian A, Berg MD, et al. 2018 American Heart Association Focused Update on Pediatric Advanced Life Support: An Update to the American Heart Association Guidelines for Cardiopulmonary Resuscitation and Emergency Cardiovascular Care. Circulation 2018;138:e731-9.

5. Kim YM, Yim HW, Jeong SH, et al. Does therapeutic hypothermia benefit adult cardiac arrest patients presenting with non-shockable initial rhythms?: A systematic review and meta-analysis of randomized and non-randomized studies. Resuscitation 2012;83:188-96.

6. Bernard SA, Gray TW, Buist MD, et al. Treatment of comatose survivors of out-of-hospital cardiac arrest with induced hypothermia. N Engl J Med 2002;346:557-63.

7. Hypothermia after Cardiac Arrest Study Group. Mild therapeutic hypothermia to improve the neurologic outcome after cardiac arrest. N Engl J Med 2002;346:549-56.

8. Nolan JP, Morley PT, Hoek TL, et al. Therapeutic hypothermia after cardiac arrest. An advisory statement by the Advancement Life support Task Force of the International Liaison committee on Resuscitation. Resuscitation 2003;57:231-5.

9. Peberdy MA, Callaway CW, Neumar RW, et al. Part 9: post-cardiac arrest care: 2010 American Heart Association Guidelines for Cardiopulmonary Resuscitation and Emergency Cardiovascular Care. Circulation 2010;122:S768-86.

10. Chen CT, Chen CH, Chen TY, et al. Comparison of in-hospital and out-of-hospital cardiac arrest patients receiving targeted temperature management: A matched case-control study. J Chin Med Assoc 2020;83:858-64.

11. Chan PS, Berg RA, Tang Y, et al. Association Between Therapeutic Hypothermia and Survival After In-Hospital Cardiac Arrest. JAMA 2016;316:1375-82.

12. Kory P, Fukunaga M, Mathew JP, et al. Outcomes of mild therapeutic hypothermia after in-hospital cardiac arrest. Neurocrit Care 2012;16:406-12.

13. Nichol G, Huszti E, Kim F, et al. Does induction of hypothermia improve outcomes after in-hospital cardiac arrest? Resuscitation 2013;84:620-5.

14. Perman SM, Grossestreuer AV, Wiebe DJ, et al. The Utility of Therapeutic Hypothermia for Post-Cardiac Arrest Syndrome Patients With an Initial Nonshockable 
Rhythm. Circulation 2015;132:2146-51.

15. Wang CH, Huang CH, Chang WT, et al. Outcomes of Adult In-Hospital Cardiac Arrest Treated with Targeted Temperature Management: A Retrospective Cohort Study. PLoS One 2016;11:e0166148.

16. Wang CJ, Yang SH, Chen CH, et al. Targeted Temperature Management for In-Hospital Cardiac Arrest: 6 Years of Experience. Ther Hypothermia Temp Manag 2020;10:153-8.

17. Peberdy MA, Kaye W, Ornato JP, et al. Cardiopulmonary resuscitation of adults in the hospital: a report of 14720 cardiac arrests from the National Registry of Cardiopulmonary Resuscitation. Resuscitation 2003;58:297-308.

18. Leong SH, Chan E, Ho BC, et al. Therapeutic

Cite this article as: Yin L, Xie D, He D, Chen Z, Guan Y, Wang J, Lin Z. Survival to hospital discharge and neurological outcomes with targeted temperature management after inhospital cardiac arrest: a systematic review and meta-analysis. Ann Palliat Med 2022;11(1):68-76. doi: 10.21037/apm-21-3403 temperature management (TTM): post-resuscitation care for adult cardiac arrest, with recommendations from the National TTM Workgroup. Singapore Med J 2017;58:408-10.

19. Scirica BM. Therapeutic hypothermia after cardiac arrest. Circulation 2013;127:244-50.

20. Abdalla M, Mohamed A, Mohamed W, et al. Targeted temperature management after cardiac arrest: Updated meta-analysis of all-cause mortality and neurological outcomes. Int J Cardiol Heart Vasc 2019;24:100400.

21. Engsig M, Søholm H, Folke F, et al. Similar long-term survival of consecutive in-hospital and out-of-hospital cardiac arrest patients treated with targeted temperature management. Clin Epidemiol 2016;8:761-8. 


\section{Appendix A Review questions according to the PICO Scheme}

* Question 1: In adults resuscitated from in-hospital cardiac arrest (P) and treated with TTM (I), survival to hospital discharge $(\mathrm{O})$

* Question 2: In adults resuscitated from in-hospital cardiac arrest (P) and treated with TTM (I), good neurological outcome, defined as grade 1 or $2(\mathrm{O})$ of brain function.

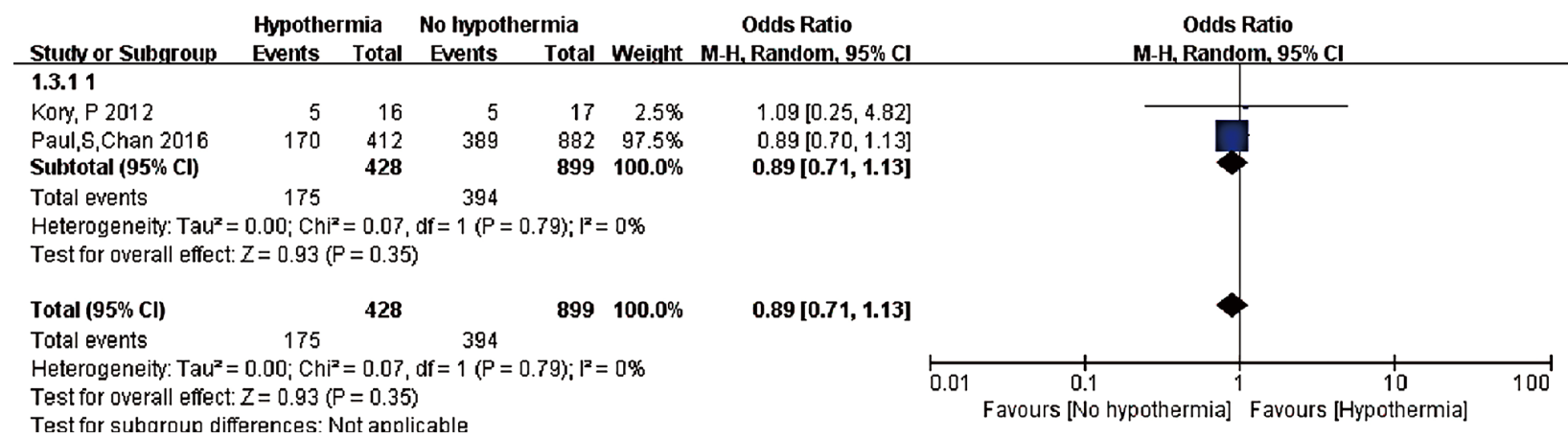

Figure S1 Survival to hospital discharge in shockable initial rhythms.

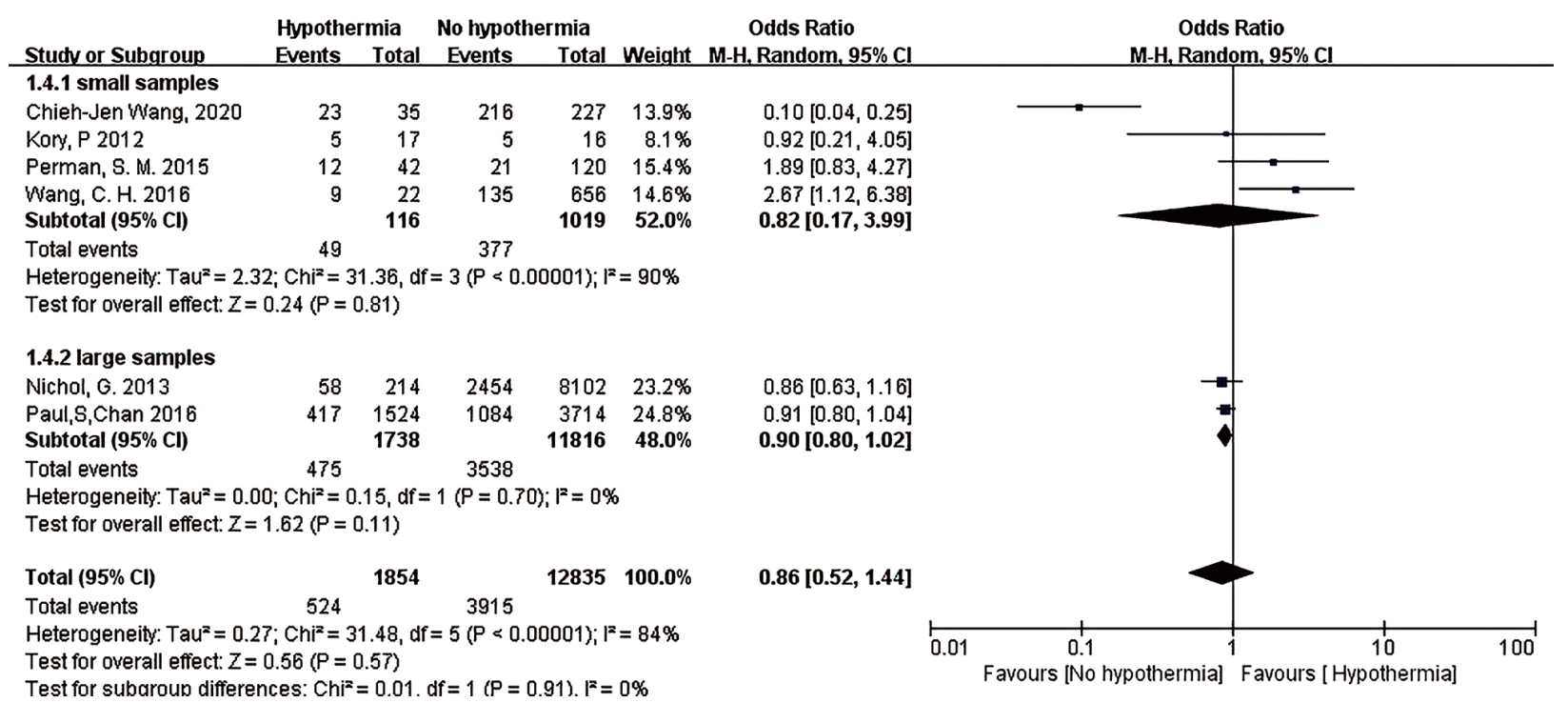

Figure S2 Survival to hospital discharge in different sample content. 


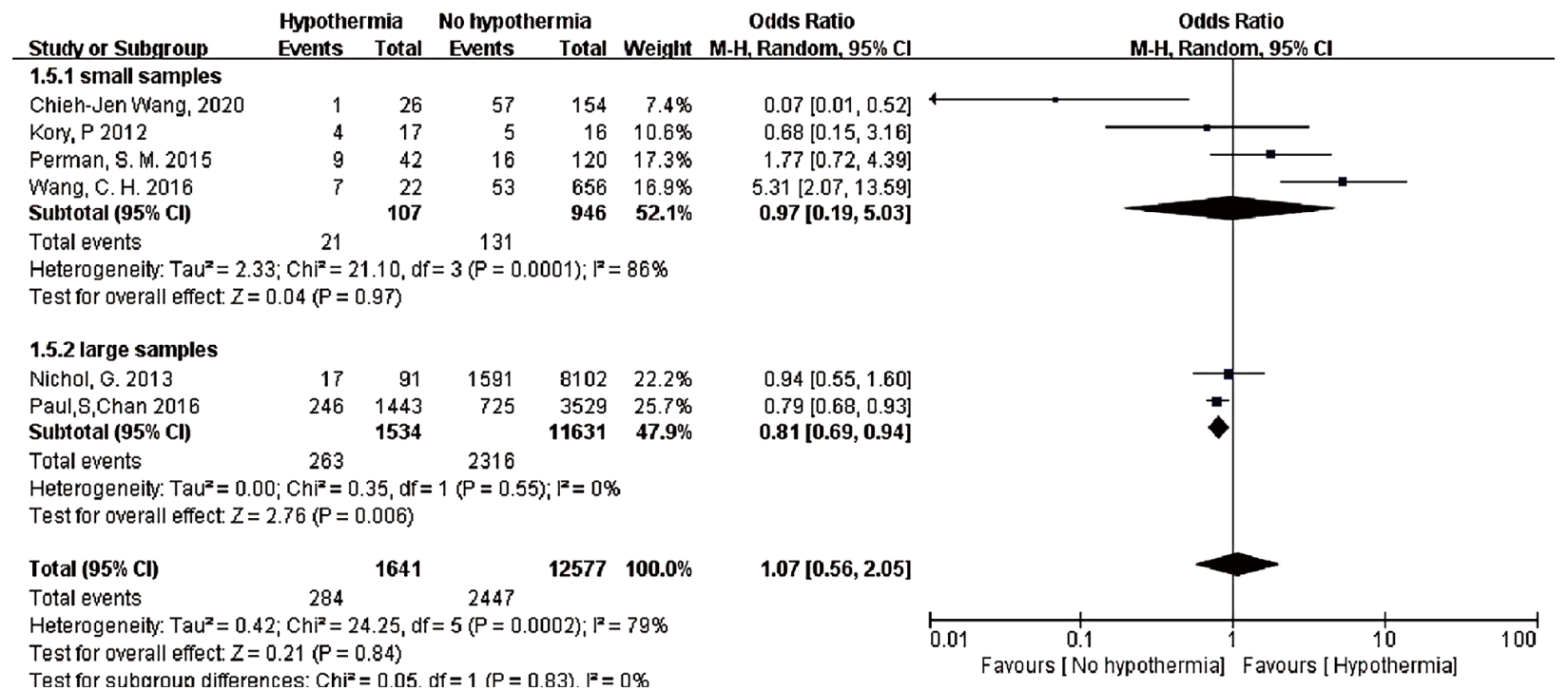

Figure S3 Good neurological outcomes in different sample content.

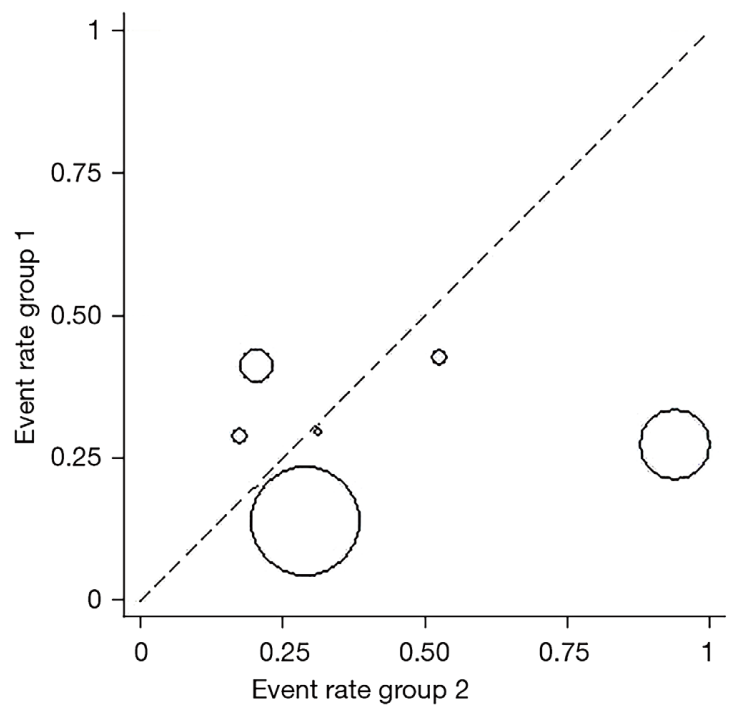

Figure S4 Survival to hospital discharge L'Abbe plot.

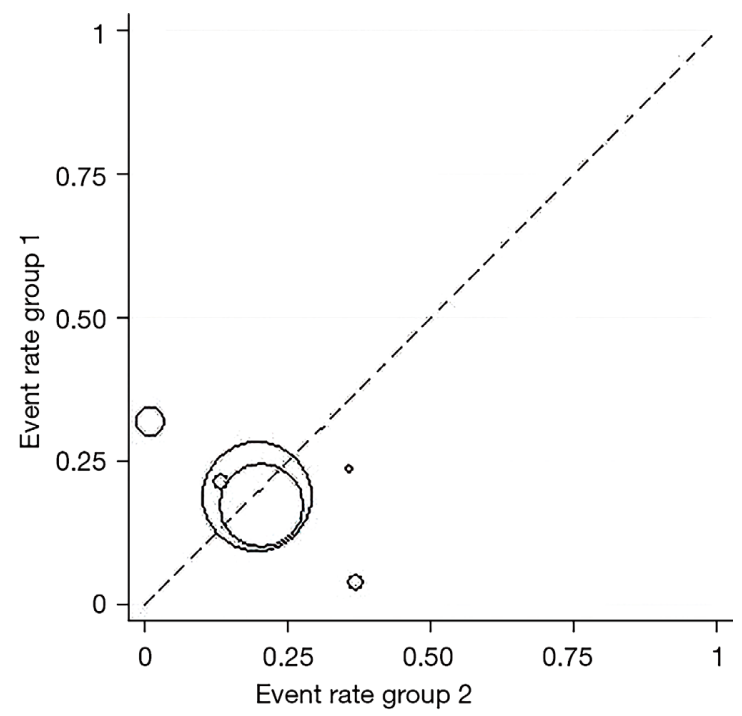

Figure S5 Good neurological outcomes L'Abbe plot. 


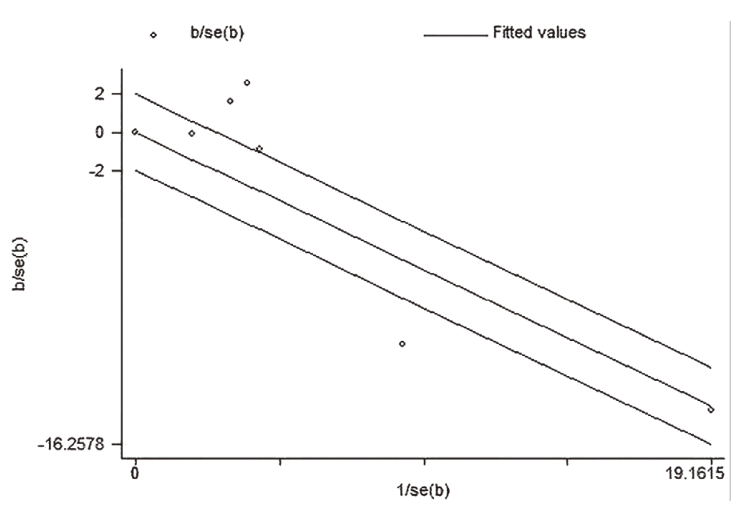

Figure S6 Survival to hospital discharge Galbraith plot.

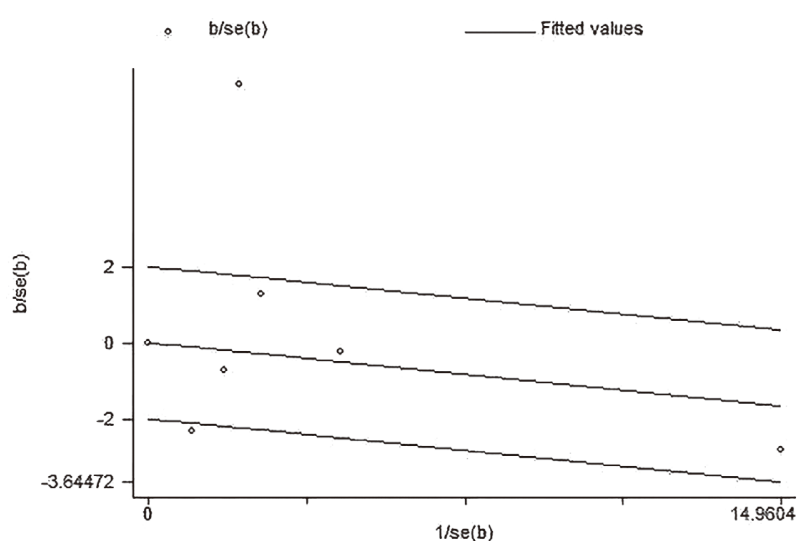

Figure S7 Good neurological outcomes Galbraith plot.

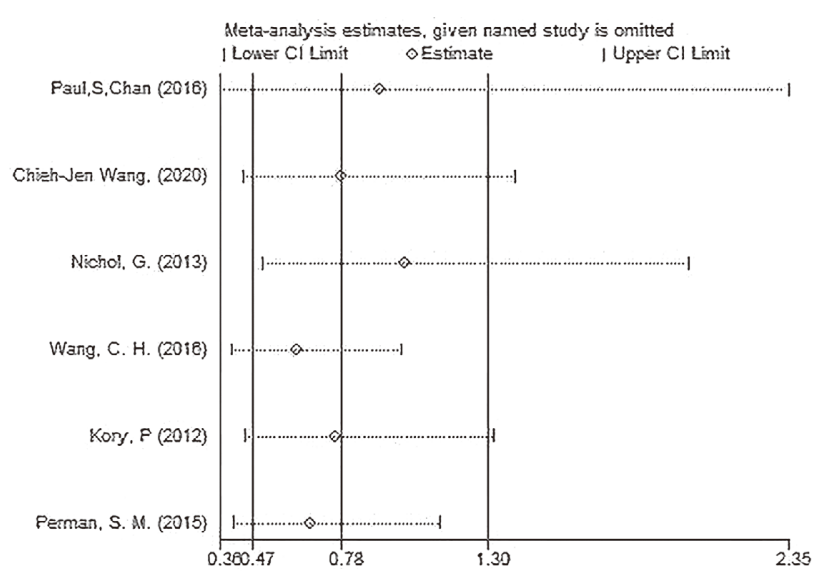

Figure S8 Impact map of a single study in the discharged survival group.

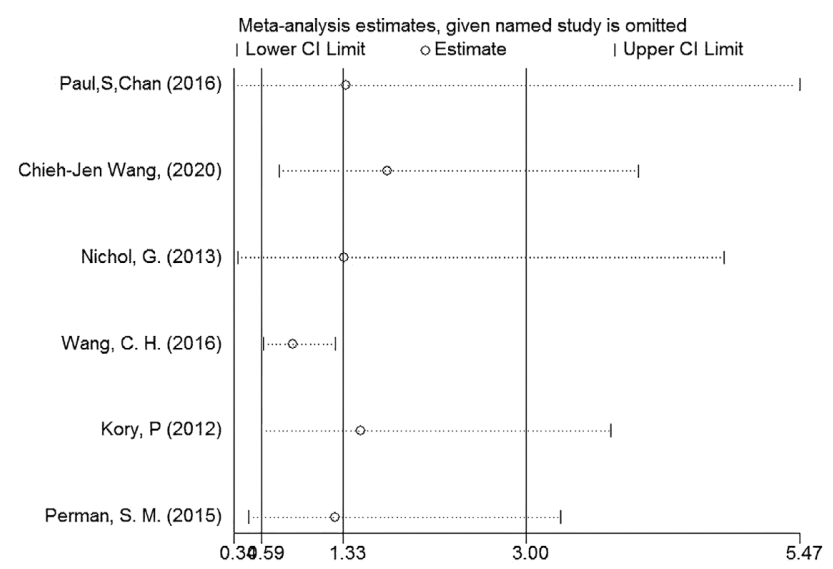

Figure S9 Impact map of a single study in the good neurological outcome group. 


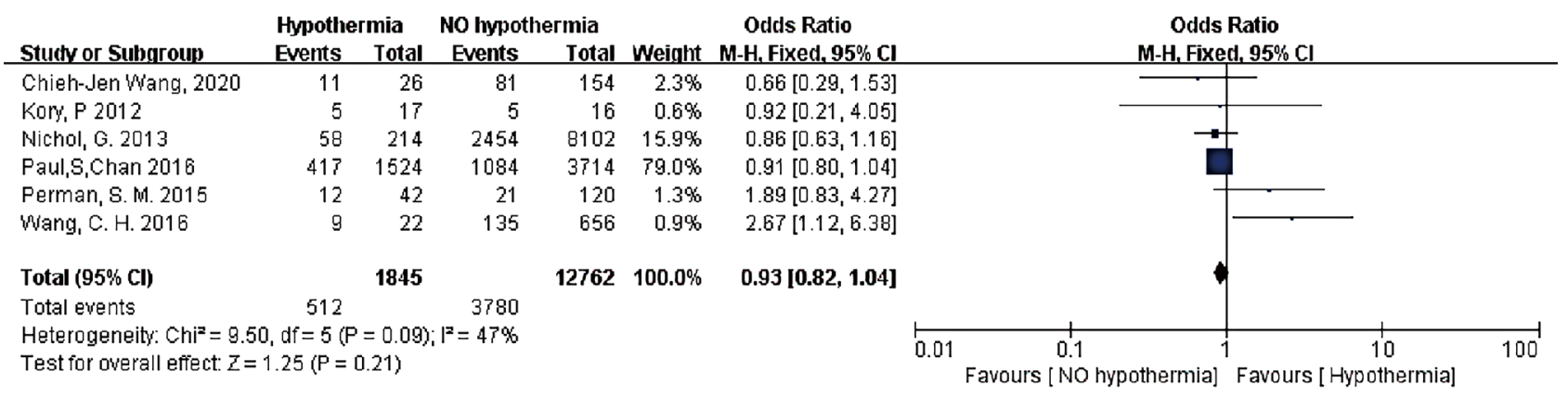

Figure S10 Randomized effect model diagram of the discharged survival group.

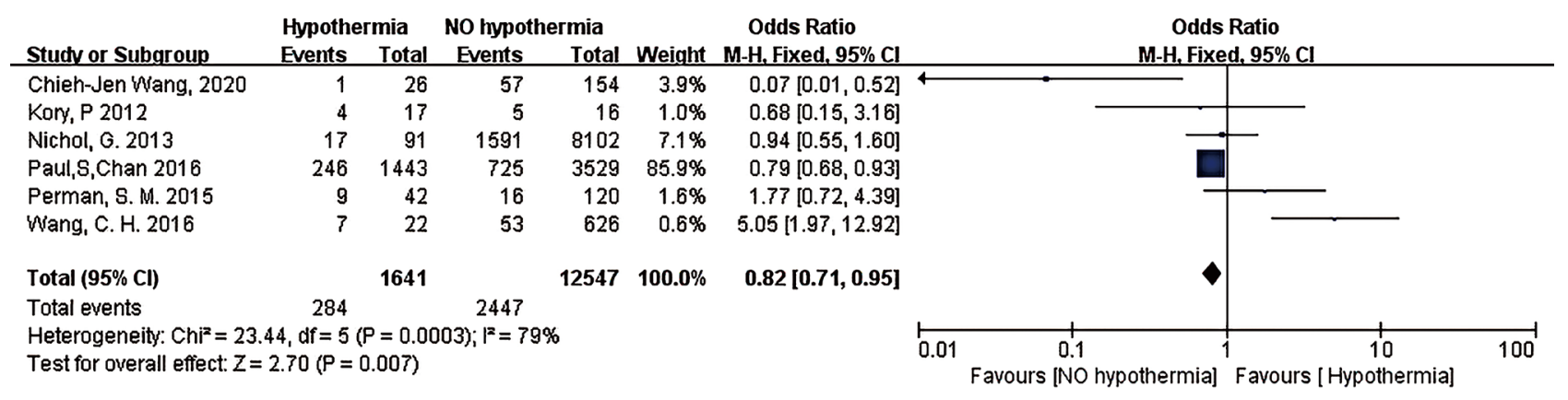

Figure S11 Neurological function randomization effect model diagram.

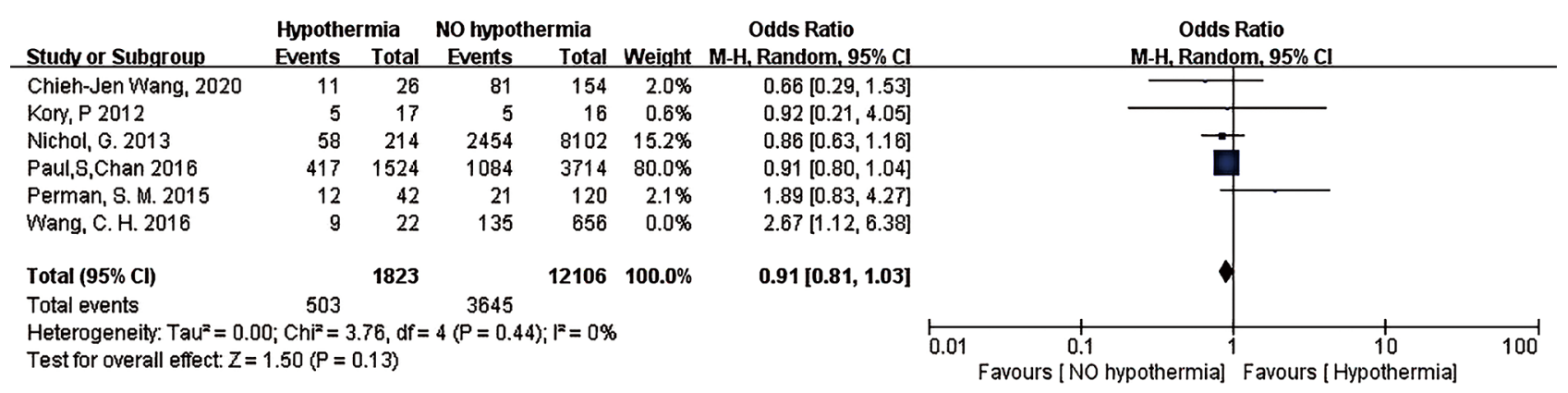

Figure S12 Single study removal impact diagram of discharge survival. 


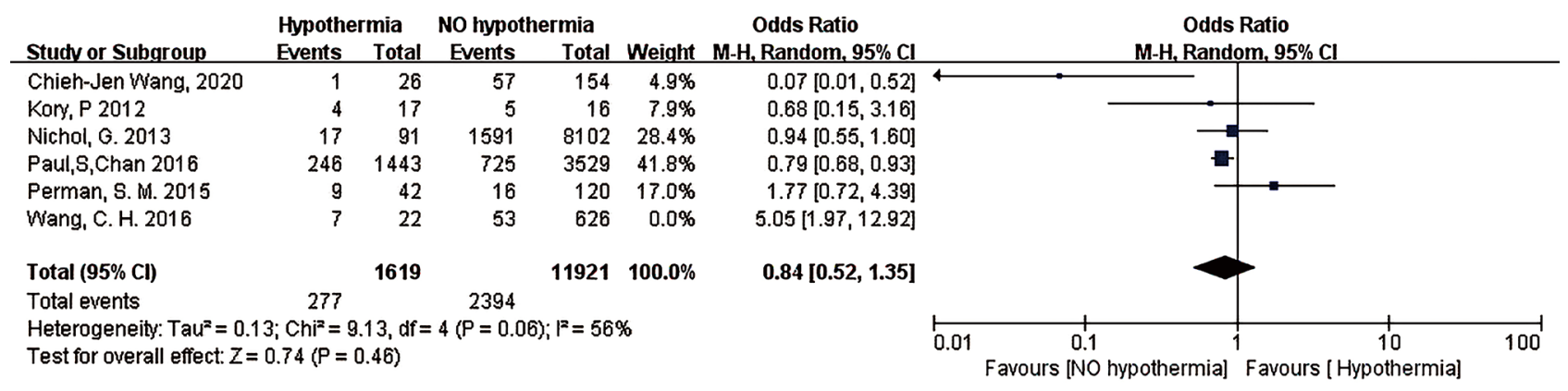

Figure S13 Single study removal impact diagram of neurological outcome.

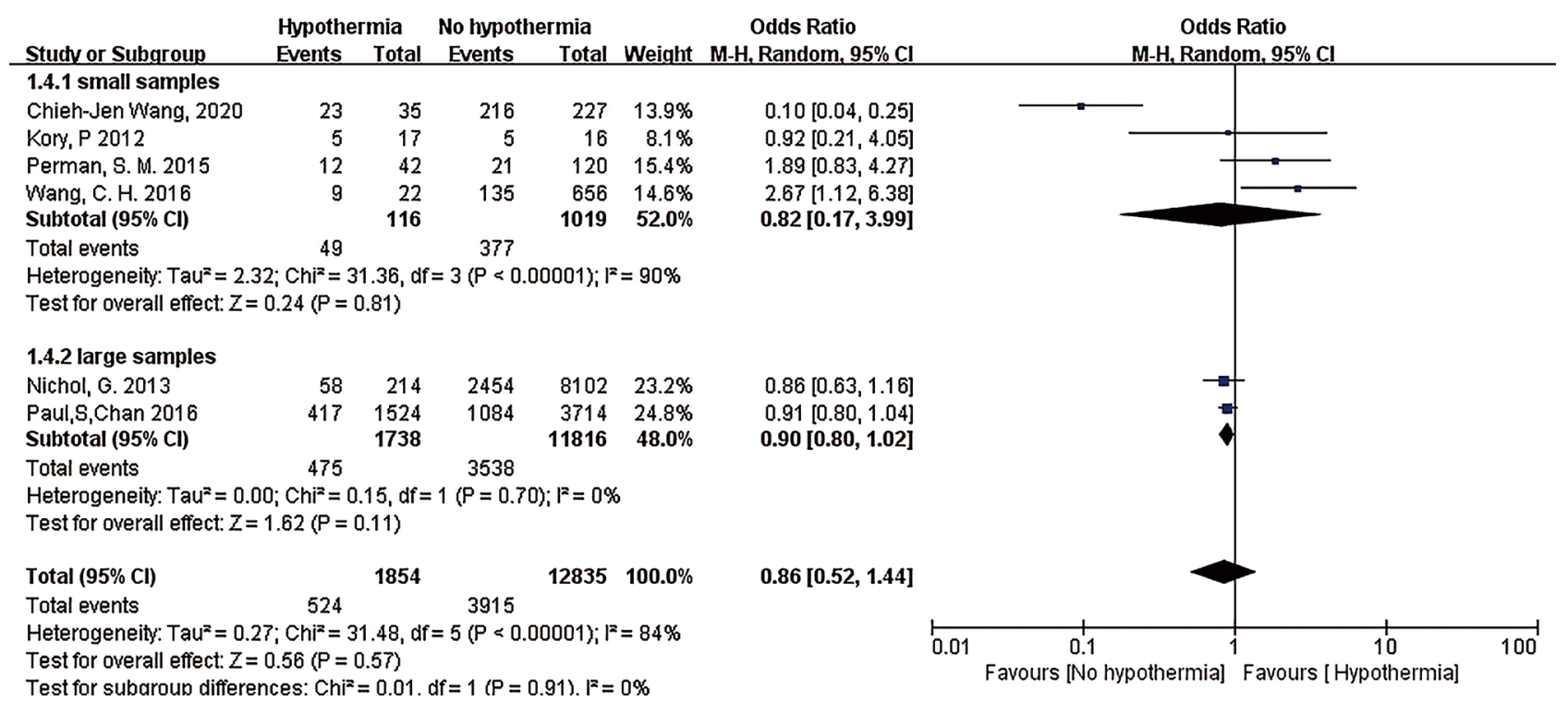

Figure S14 Funnel chart. 